\title{
COVID-19 pneumonia: increased choline uptake with 18F-choline PET/CT
}

\author{
Laura Olivari $^{1}$ (D) $\cdot$ Niccolò Riccardi $^{2} \cdot$ Paola Rodari $^{2} \cdot$ Andrea Angheben $^{2} \cdot$ Paolo Artioli $^{3} \cdot$ Matteo Salgarello $^{1}$
}

Received: 28 April 2020 / Accepted: 13 May 2020 / Published online: 4 June 2020

(C) Springer-Verlag GmbH Germany, part of Springer Nature 2020

Even if challenging, early identification of the acute respiratory disease (ARDS) held by the novel coronavirus, SARS$\mathrm{CoV}-2$, is crucial for patient clinical management.

We report the case of a 59-year-old man with biochemical recurrence of prostate cancer (prostate-specific antigen PSA $12 \mathrm{ng} / \mathrm{ml}$ ) after radical prostatectomy held in September 2019 (pre-surgery PSA 4 ng/ml, Gleason score $4+3$, negative bone scan) performing choline-PET (18F-choline positron emission tomography) to assess disease spread.

The patient arrived to our department with no fever, no shortness of breath, no fatigue or myalgia, no cough, or diarrhea. He had no medical history of pulmonary diseases, normal respiratory rate, and $\mathrm{SpO}$. Choline-PET revealed prostate cancer bone involvement with no pathological lymph nodes in pelvic area. Indeed, the exam showed bilateral subsegmental peripheral areas of ground glass opacities (GGO) in the lungs. All the pulmonary lesions presented an increased choline-PET uptake (SUV max range 3-4) without pleural and mediastinal lymph nodes involvement (Fig. 1). The asymptomatic patient was quarantined and isolated at home as COVID-19 possible case. Within $36 \mathrm{~h}$ after the choline-PET, the patient developed dizziness, fatigue, and respiratory failure, leading to hospitalization. SARS-CoV-2 nucleid testing resulted positive on both nasal and pharyngeal swabs.

This article is part of the Topical Collection on Image of the month

Laura Olivari

laura.olivari@sacrocuore.it

\section{Niccolò Riccardi}

niccolo.riccardi@sacrocuore.it

Paola Rodari

paola.rodari@sacrocuore.it

Andrea Angheben

andrea.angheben@sacrocuore.it

Paolo Artioli

paolo.artioli@studenti.unipd.it
Matteo Salgarello

matteo.salgarello@sacrocuore.it

1 Department of Nuclear Medicine, IRCCS Ospedale Sacro Cuore Don Calabria, Via Don Angelo Sempreboni, 5- Negrar di Valpolicella, Verona, Italy

2 Department of Infectious, Tropical Diseases and Microbiology, IRCCS Sacro Cuore Don Calabria Hospital, Negrar di Valpolicella, Verona, Italy

3 Nuclear Medicine, University of Padova, Padova, Italy 

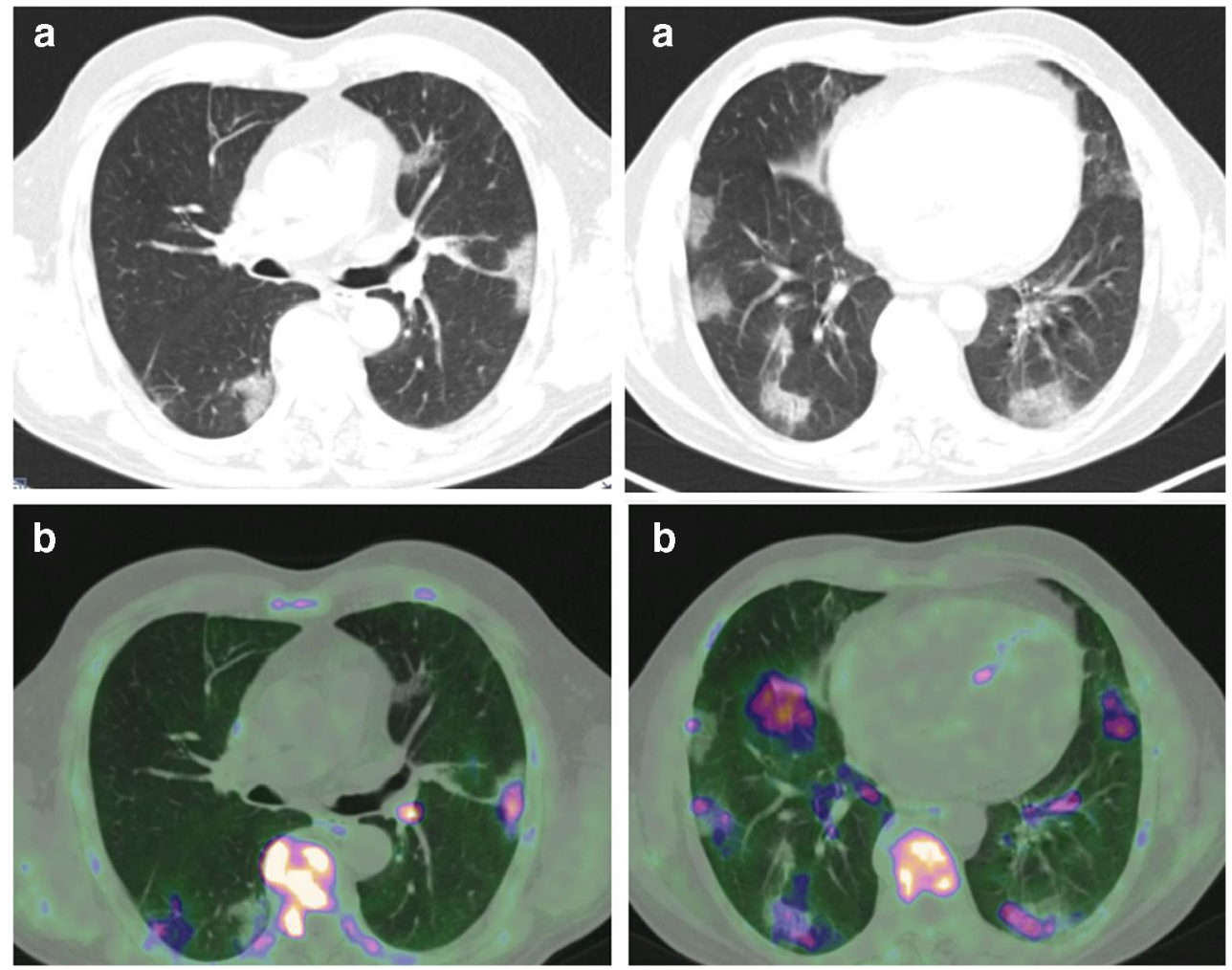

Co-registered computerized tomography (CT) is more sensible in reviling lung involvement before clinical symptoms' appearance in SARS-CoV-2 disease [1,2]. A previous study described the potential role of FDG-PET in the evaluation of patients with SARS-CoV-2 infection [3]. Remarkably, in our case, all the pulmonary lesions showed an increased choline uptake reflecting high macrophage inflammatory burden [4]. These findings suggest that pneumonia related to macrophage activation syndrome may be an important feature of SARSCoV-2 disease [5]. Choline-PET may identify patients about to develop SARS-CoV-2-related ARDS.

Acknowledgments Our Ethics Committee acknowledged and authorized the publication.

Data sharing No datasets were generated or analyzed during the current study.

\section{Compliance with ethical standards}

Conflict of interest The authors declare that they have no conflicts of interest.

Ethical approval This article does not contain any studies with animals performed by any of the authors. All procedures performed in studies involving human participants were in accordance with the ethical standards of the institutional and/or national research committee and with the principles of the 1964 Declaration of Helsinki and its later amendments or comparable ethical standards.
Statement of informed consent Informed consent to assess PETcholine was obtained from the patient included in the study. The patient performed choline-PET to assess disease spread in biochemical recurrence of prostate cancer (PC) after complete prostatectomy. The patient gave a written consent for the case report publication in fully anonymized form.

\section{References}

1. Shi H, Han X, Jiang N, et al. Radiological findings from 81 patients with COVID-19 pneumonia in Wuhan, China: a descriptive study. Lancet Infect Dis. 2020;20(4):425-34. https://doi.org/10.1016/ S1473-3099(20)30086-4

2. Lee EYP, Ng MY, Khong PL. COVID-19 pneumonia: what has CT taught us? Lancet Infect Dis. 2020;20(4):384-5. https://doi.org/10. 1016/S1473-3099(20)30134-1.

3. Deng Y, Lei L, Chen Y, Zhang W. The potential added value of FDG PET/CT for COVID-19 pneumonia [published online ahead of print, 2020 Mar 21]. Eur J Nucl Med Mol Imaging. 2020:1-2. https://doi. org/10.1007/s00259-020-04767-1.

4. Snider SA, Margison KD, Ghorbani P, et al. Choline transport links macrophage phospholipid metabolism and inflammation. J Biol Chem. 2018;293(29):11600-11. https://doi.org/10.1074/jbc. RA118.003180.

5. Pedersen SF, Ho YC. SARS-CoV-2: a storm is raging. J Clin Invest. 2020;130(5):2202-5. https://doi.org/10.1172/JCI137647.

Publisher's note Springer Nature remains neutral with regard to jurisdictional claims in published maps and institutional affiliations. 